\title{
Generation of Zonal Flow and Magnetic Field by Planetary Waves in the Earth's Ionosphere
}

\author{
T. D. Kaladze1, Kh. Chargazia',2, 0. Kharshiladze',3, L. V. Tsamalashvili1 \\ ${ }^{1}$ I. Vekua Institute of Applied Mathematics, Tbilisi State University, Tbilisi, Georgia \\ ${ }^{2} \mathrm{M}$. Nodia Institute of Geophysics, Tbilisi State University, Tbilisi, Georgia \\ ${ }^{3}$ Faculty of Exact and Natural Sciences, Tbilisi State University, Tbilisi, Georgia \\ Email: tamaz_kaladze@yahoo.com
}

Received 26 December 2015; accepted 26 February 2016; published 29 February 2016

\begin{abstract}
Possibility of generation of large-scale sheared zonal flow and magnetic field by coupled under the typical ionospheric conditions short-scale planetary low-frequency waves is shown. Propagation of coupled internal-gravity-Alfven, Rossby-Khantadze, Rossby-Alfven-Khantadze and collision-less electron skin depth order drift-Alfven waves is revealed and investigated in detail. To describe the nonlinear interaction of such coupled waves with sheared zonal flow the corresponding nonlinear equations are deduced. The instability mechanism is based on the nonlinear parametric triple interaction of the finite amplitude short-scale planetary waves leading to the inverse energy cascade toward the longer wavelengths. It is shown that under such interaction intense sheared magnetic fields can be generated. Appropriate growth rates are discussed in detail.
\end{abstract}

\section{Keywords}

Planetary Waves, Sheared Zonal Flow, Ionosphere, Sheared Magnetic Field

\section{Introduction}

Numerous ground-based and satellite experiments justify permanent existence of zonal flows in different layers of the Earth's atmosphere [1] [2]. Such sheared flows have inhomogeneous velocity along the meridians and as a rule are accompanied by ultra-low-frequency (ULF) planetary-scale perturbations in the different layers of the Earth's ionosphere [2]-[6]. Due to the decisive influence on the global atmospheric circulation Rossby-type perturbations attract the special attention [7] [8]. Interaction of the sheared zonal flow with the nonlinear vortical formations having the different geophysical applications has been studied in [9]. Dynamical interaction of the sheared zonal flow with Rossby solitary vortical formations in the Earth's atmosphere and ionosphere have been studied both analytically and numerically in [10] [11]. Such investigation shows that the zonal flow energy accumulates into the vortical structures leading to partitioning into several pieces composing a turbulent state.

Latitudinal inhomogeneity of both the Coriolis parameter and the geomagnetic field, and also caused by the

How to cite this paper: Kaladze, T.D., Chargazia, Kh., Kharshiladze, O. and Tsamalashvili, L.V. (2016) Generation of Zonal Flow and Magnetic Field by Planetary Waves in the Earth's lonosphere. Journal of Applied Mathematics and Physics, 4, 487491. http://dx.doi.org/10.4236/jamp.2016.42054 
gravitation so called stratification under the ionospheric conditions lead to the coupling of different electromagnetic (EM) planetary waves such as coupled Rosssby-Khantadze (CRK) [12] [13], Internal-Gravity-Alfven (CIGA) [14], Rossby-Alfven-Khantadze modes (CRAK) [15] [16] and drift-Alfven modes (CDA) [17] [18].

According to the nowadays presentations sheared zonal flows are created by the nonhomogeneous heating of the atmospheric layers by solar radiation. But during the last decade a great amount of publications revealed different nonlinear mechanisms leading to the generation of sheared zonal flow. Namely the excitation of zonal flow based on the triple planetary waves nonlinear parametric instability in the Earth's ionospheric medium was widely investigated (see [14] [16]-[30] and references cited in).

In the given paper we review the possibility of generation of zonal flow and magnetic field by different coupled EM ULF waves in the weakly ionized Earth's ionosphere. The local Cartesian system of coordinates $(x, y, z)$ is used, where $x$-axis is directed from the west to the east, the $y$-axis is directed from the south to the north and the $z$-axis coincides with the local vertical direction. We show that the turbulence of such short-wavelength coupled waves may be unstable with the excitation of low-frequency and large-scale perturbation of the sheared zonal flow and sheared magnetic field. The nonlinear mechanism of the instability is driven by the advection of vorticity and is based on the parametric excitation of zonal flow by three finite-amplitude coupled modes leading to the inverse energy cascade toward the longer wavelength. The corresponding driving forces are stipulated by the Reynolds and Maxwell's stresses.

\section{Generation by CDA Waves}

The generation of large-scale zonal flows and magnetic fields due to small-scale drift-Alfven turbulence in the ionospheric plasma medium is investigated (see details in [18]). System of two nonlinear 3D equations determining electrostatic and vector potentials describing the dynamics of the wave structures with finite ion Larmor radius is obtained. Note that they are valid for the structures till the skin scale. The optimal growth rate of the zonal modes $\gamma \sim c q_{x}^{2} E / k_{x} B_{0}$, where $E$ is generated electric field stress, $B_{0}$ is the value of the Earth's geomagnetic field, $q_{x}$ is the zonal flow wave number, $k_{x}$ is the pumping wave number, such that $q_{x} / k_{x} \ll 1$. For the characteristic ionospheric parameters $E \sim 10^{-2} \mathrm{v} / \mathrm{m}, B_{0} \sim 3 \times 10^{-5} \mathrm{~T}, q_{x} \sim 0.01 k_{x}$, so we get $\gamma \sim 10^{5} \mathrm{~s}^{-1}$. Thus, the considered parametric instability pumps the energy of initial CDA waves into the energy of large-scale zonal flow and EM fields in $10^{-5} \mathrm{~s}$.

\section{Generation by CRK Waves}

It is shown that in the Earth's E-layer under the prevalent Hall conductivity due to the latitudinal non-homogeneity of geomagnetic field, slow and fast waves can couple and form EM CRK waves (see details in [12] [13] [26] [27] [29] [30]). A linear dispersion relation for the CRK waves is obtained. A simplified set of 2D nonlinear equations describing the dynamics of CRK modes is deduced. For zonal flows as well as magnetic field nonlinear generation problem, a modified parametric technique is used and primary mode spectrum assumed to be arbitrary. It is shown that driven by the EM CRK turbulence, zonal flows propagate along the geographical parallels. The corresponding flow velocity depends only on meridional $y$-coordinates. It is found that the maximum zonal growth rate is achieved when wave number $k_{y}=0$. Under the E-layer conditions the following zonal growth rate numerical value $\gamma \sim 10^{-7} s^{-1}$ is obtained. For CRK pumping waves the mean magnetic field is of the order of $q_{y}^{2}$ with respect to excited mean zonal flow and is caused through the latitudinal non-homogeneity of the Earth's stationary magnetic field. The excited magnetic field is in local vertical z-direction whereas zonal flow is sheared in the meridional y-direction. The maximum numerical value for generated mean magnetic field is $\approx 10^{3} n T$.

\section{Generation by CIGA Waves}

The basic 2D nonlinear equations describing the dynamics of zonal flow generation and coupled internal-gravity and Alfven wave propagation under the Boussinesq approximation are constructed (see details in [14] [22] [28] [30]). Caused by the gravity force action altitude distribution of the equilibrium density and pressure of the medium (so called stratification) is taken into account. A linear dispersion relation for the CIGA waves is obtained. Taking into account the EM character of CIGA waves the existence of the new coupled waves propagating along the parallels in the ionospheric E-layer is shown. This shows a mixture of ordinary internal-gravity waves 
and a new dispersive Alfven wave. It is shown that internal-gravity and Alfven waves can be coupled through the $z$-component of the wavevector $\left|k_{z}\right| \sim \omega_{g} /\left(B_{0 z} / \mu_{0} \rho(0)\right)$. For the ionospheric E-layer parameters $B_{0} \approx 0.5 \times 10^{-4} \mathrm{~T}$ and mass density $\rho=\left(10^{-7}-10^{-8}\right) \mathrm{kgm}^{-3}$ we get that the squared Alfven velocity $\mathrm{v}_{A}^{2}=\left(10^{4}-10^{5}\right) \mathrm{m}^{2} \mathrm{~s}^{-2}$; for the density scale height $H=10 \mathrm{~km}$ the Brunt-Väisälä frequency $\omega_{g}=(g / H)^{1 / 2} \approx 10^{-2} \mathrm{~s}^{-1}$. So numerically we get $\left|k_{z}\right| \sim 10^{-4} \mathrm{~m}^{-1}$. Typical values of phase velocities for the internal-gravity waves is $(10-20) \mathrm{ms}^{-1}$ and $5-50 \mathrm{~km}$ is the typical values of the corresponding wavelengths. Dealing with zonal flow excitation, we used the parametric approach allowing one to consider monochromatic primary modes. The investigated sheared zonal flow propagates along the Earth's parallels. The corresponding velocity is only dependent on the vertical $z$-coordinate. Excited mean magnetic field is in local vertical straight $z$-direction; the zonal flow is also sheared in this direction. The maximum growth rate for zonal flow is estimated $\gamma \sim 10^{-4} \mathrm{~s}^{-1}$. As to the excited magnetic field it is of $10^{2} \mathrm{nT}$.

\section{Generation by CRAK Waves}

Nonlinear dynamics of CRAK EM planetary modes in the ionospheric E-layer is investigated (see details in [16] [30]). It is shown that under the prevalent action of Hall conductivity, owing to the latitudinal non-homogeneity of both the Coriolis parameter and stationary geomagnetic field, a new type of CRAK wave can propagate. In contrast to CRK and CIGA investigations, the perturbed magnetic field of other polarization (with $x$ - and $y$-components) is considered. This makes the problem essentially spatially three-dimensional. As a result, owing to the existence of magnetic field perturbations, Alfven waves also became incorporated. The action of these effects leads to the coupled propagation of EM CRAK modes. A simplified set of spatially 3D nonlinear equations describing the dynamics of EM CRAK modes is obtained. The linear dispersion relation for the EM CRAK mode is given. Under such coupling, a new type of dispersive Alfven waves is revealed. Using the modified parametric approach, the possibility of large-scale zonal flow and magnetic field generation by relatively shortscale EM CRAK modes in the weakly ionized ionospheric E-layer gas is demonstrated. Nonlinear instability of short-wavelength turbulence of CRAK EM planetary modes with respect to the excitation of low-frequency and large-scale perturbations of sheared zonal flow and magnetic field is revealed. Generated by the EM CRAK modes, zonal flows propagate along geographical parallels and the corresponding flow velocity depends only on meridional $y$-coordinates. The maximum growth rate is achieved at $k_{y}=0$, when the group velocity $V_{g}=0$ and consequently the real part of the oscillations for zonal flow becomes zero. Here zonal flow is only contributed by Rossby waves and the corresponding growth rate is obtained. The following zonal growth rate value $\gamma \approx 10^{-1} s^{-1}$ is obtained. Here the mean magnetic field excitation requires special attention. The generated magnetic field is of the order of $q_{y}^{2}$ with respect to the excited mean zonal flow and is caused only by the existence of Alfven waves. The excited mean magnetic field has prevalent component $\delta B_{y}$ and the zonal flow is sheared in the meridional $y$-direction. Under the values of the ionospheric parameters numerically $\left|\delta B_{y}\right|=\left(10^{2}-10^{3}\right) n T$.

\section{Conclusion}

Possibility of the generation of sheared zonal flow and sheared magnetic field in the weakly ionized conductive ionospheric gas is investigated. Propagation of electromagnetic coupled drift-Alfven (CDA), Rossby-Khantadze (CRK), Internal-gravity-Alfven (CIGA), and Rossby-Alfven-Khantadze (CRAK) waves is analyzed. It is shown that owing to the latitudinal nonhomogeneity of the geomagnetic field slow and fast waves can couple and form CRK waves. It is shown that Internal-gravity and Alfven waves can be coupled through the $z$-component of wavevector. In contrast to CRK and CIGA waves the perturbed magnetic field of other polarization (with $x$ - and $y$-components) is considered for CRAK and CDA waves. This makes the problem essentially three dimensional. As a result, owing to the existence of the magnetic field perturbations, Alfven waves also became incorporated. Action of these effects leads to the coupled propagation of EM coupled Rossby-Alfven-Khantadze and driftAlfven modes.

Simplified set of nonlinear equations describing the dynamics of mentioned coupled EM modes is obtained: 1) spatially 2D equations in case of CRK and CIGA and 2) spatially 3D equations in case of CRAK and CDA modes, respectively.

Nonlinear instability of short wavelength turbulence of mentioned CIGA, CRK, CRAK, and CDA EM planetary modes with respect to the excitation of low-frequency and large-scale perturbations of sheared zonal flow and magnetic field is revealed. The nonlinear mechanism of the instability is driven by the advection of vorticity 
and is based on the parametric excitation of zonal flow by three finite-amplitude coupled modes leading to the inverse energy cascade toward the longer wavelength. The growth rates of the corresponding instability and the conditions for driving them are determined. The possibility of generation of the intense mean magnetic field is shown.

Obtained results can be used to elucidate different laboratory and space experiments.

\section{References}

[1] Petviashvili, V.I. and Pokhotelov, O.A. (1992) Solitary Waves in Plasmas and in the Atmosphere. Gordon \& Breach, Reading.

[2] Kamide, Y. and Chian, A. (Editors) (2004) Handbook of the Solar-Terrestrial Environment. Springer.

[3] Kelley, M.C. (1989) The Earth’s Ionosphere, Plasma Physics and Electrodynamics. Academic Press, Inc., San Diego, California.

[4] Alperovich, L.S. and Fedorov, E.N. (2007) Hydromagnetic Waves in the Magnetosphere and the Ionosphere. Springer. http://dx.doi.org/10.1007/978-1-4020-6637-5

[5] Kaladze, T.D., Pokhotelov, O.A., Sagdeev, R.Z., Stenflo, L. and Shukla, P.K. (2003) Planetary Electromagnetic Waves in the Ionospheric E-layer. Journal Atmospheric and Solar-Terrestrial Physics, 65, 757-764. http://dx.doi.org/10.1016/S1364-6826(03)00042-7

[6] Khantadze, A.G., Jandieri, G.V., Ishimaru, A., Kaladze, T.D., and Diasamidze, Zh.M. (2010) Electromagnetic Oscillations of the Earth's Upper Atmosphere (Review). Annales Geophysicae, 28, 1387-1399. http://dx.doi.org/10.5194/angeo-28-1387-2010

[7] Satoh, M. (2004) Atmospheric Circulation Dynamics and General Circulation Models. Springer.

[8] Kaladze, T.D., Aburjania, G.D., Kharshiladze, O.A., Horton, W. and Kim, Y.-H. (2004) Theory of Magnetized Rossby Waves in the Ionospheric E Layer. Journal of Geophysical Research, 109, A05302 (1-14).

[9] Marcus, P.S., Kundu, T. and Changhoon Lee (2000) Vortex Dynamics and Zonal Flows. Physics of Plas-mas, 7, 1630-1640. http://dx.doi.org/10.1063/1.874045

[10] Kaladze, T.D., Wu, D.J., Tsamalashvili, L.V. and Jandieri, G.V. (2007) Localized Magnetized Rossby Structures under Zonal Shear Flow in the Ionospheric E-Layer. Physics Letters A, 365, 140-143. http://dx.doi.org/10.1016/j.physleta.2007.01.002

[11] Kaladze, T.D., Pokhotelov, O.A., Stenflo, L., Rogava, J., Tsamalashvili, L.V. and Tsiklauri, M. (2008) Zonal Flow Interaction with Rossby Waves in the Earth's Atmosphere: A Numerical Simulation. Physics Letters A, 372, 5177-5180. http://dx.doi.org/10.1016/j.physleta.2008.06.008

[12] Kaladze, T.D., Tsamalashvili, L.V. and Kahlon, L.Z. (2011) Rossby-Khantadze Electromagnetic Planetary Vortical Motions in the Ionospheric E-layer. Journal of Plasma Physics, 77, 813-828. http://dx.doi.org/10.1017/S0022377811000237

[13] Futatani, S., Horton, W. and Kaladze, T.D. (2013) Nonlinear Propagation of Rossby-Khantadze Electro-magnetic Planetary Waves in the Ionospheric E-Layer. Physics of Plasmas, 20, 102903 (1-12).

[14] Kaladze, T.D., Kahlon, L.Z., Tsamalashvili, L.V. and Kaladze, D.T. (2012) Generation of Zonal Flow and Magnetic Field by Coupled Internal-Gravity and Alfven Waves in the Ionospheric E-layer. Journal of Atmospheric and Solar-Terrestrial Physics, 89, 110-119. http://dx.doi.org/10.1016/j.jastp.2012.08.012

[15] Kaladze, T.D. and Tsamalashvili, L.V. (2001) Nonlinear Alfven-Rossby Vortical Structures in the Earth's Ionosphere. Physics Letters A, 287, 137-142. http://dx.doi.org/10.1016/S0375-9601(01)00459-5

[16] Kaladze, T.D., Horton, W., Kahlon, L.Z., Pokhotelov, O. and Onishchenko, O. (2013) Zonal Flows and Magnetic Fields Driven by Large-Amplitude Rossby-Alfven-Khantadze Waves in the E-layer Ionosphere. Journal of Geophysical Research: Space Physics, 118, 7822-7833. http://dx.doi.org/10.1002/2013ja019415

[17] Kaladze, T.D., Wu, D.J. and Yang, L. (2007) Small-scale Drift-Alfven Wave Driven Zonal Flows in Plasmas. Physics of Plasmas, 14, 032305 (1-8).

[18] Aburjania, G.D., Chargazia, Kh.Z., Zelenyi, L.M. and Zimbardo, G. (2009) Large-Scale Zonal Flow and Magnetic Field Generation Due to Drift-Alfven Turbulence in Ionospheric Plasma. Planetary and Space Science, 57, 1474-1484. http://dx.doi.org/10.1016/j.pss.2009.07.007

[19] Kaladze, T.D., Wu, D.J., Pokhotelov, O.A., Sagdeev, R.Z., Stenflo, L. and Shukla, P.K. (2005) Drift Wave Driven Zonal Flows in Plasmas. Physics of Plasmas, 12, 122311 (1-6).

[20] Kaladze, T.D., Wu, D.J., Pokhotelov, O.A., Sagdeev, R.Z., Stenflo, L. and Shukla, P.K. (2007) Zonal Flow Generation by Magnetized Rossby Waves in the Ionospheric E-Layer. Proceedings of the 12th Regional Conference on Mathe- 
matical Physics, Islamabad, Pakistan, 27 March-1 April 2006, 237-251. (Eds. M. Jamil Aslam, Faheem Hussain, Asghar Qadir, Riazuddin, Hamid Saleem, World Scientific Publishing)

[21] Kaladze, T.D., Wu, D.J., Pokhotelov, O.A., Sagdeev, R.Z., Stenflo, L. and Shukla, P.K. (2007) Ross-by-Wave Driven Zonal Flows in the Ionospheric E-layer. Journal of Plasma Physics, 73, 131-140. http://dx.doi.org/10.1017/S0022377806004351

[22] Horton, W., Kaladze, T.D., Van Dam, J.W. and Garner, T.W. (2008) Zonal Flow Generation by Internal Gravity Waves in the Atmosphere. Journal of Geophysical Research, 113, A08312 (1-8).

[23] Kaladze, T.D., Shah, H.A., Murtaza, G., Tsamalshvili, L.V., Shad, M. and Jandieri, G.V. (2009) Influence of NonMonochromaticity on Zonal-Flow Generation by Magnetized Rossby Waves in the Ionospheric E-Layer. Journal of Plasma Physics, 75, 345-357. http://dx.doi.org/10.1017/S0022377808007678

[24] Kaladze, T.D., Pokhotelov, O.A. and Shad, M. (2010) Drift Wave Driven Zonal Flows in Electron-Positron-Ion Plasmas. Journal of Plasma Physics, 76, 635-643. http://dx.doi.org/10.1017/S0022377809990869

[25] Kaladze, T.D., Shad, M. and Tsamalashvili, L.V. (2011) Generation of Zonal Flow in the Earth's Dissipative Ionospheric F-Layer. Physics Letters A, 375, 2637-2638. http://dx.doi.org/10.1016/j.physleta.2011.05.058

[26] Kaladze, T.D., Kahlon, L.Z. and Tsamalashvili, L.V. (2012) Excitation of Zonal Flow and Magnetic Field by RossbyKhantadze Electromagnetic Planetary Waves in the Ionospheric E-layer. Physics of Plasmas, 19, 022902 (1-12).

[27] Kaladze, T.D., Kahlon, L.Z., Horton, W., Pokhotelov, O. and Onishchenko, O. (2014) Shear Flow Driven Rossby-Khantadze Electromagnetic Planetary Vortices in the Ionospheric E-Layer. Europhysics Letters, 106, 29001 (1-5).

[28] Onishchenko, O., Pokhotelov, O., Horton, W., Smolyakov A., Kaladze, T. and Fedun, V. (2014) Rolls of the Internal Gravity Waves in the Earth's Atmosphere. Annales Geophysicae, 32, 181-186. http://dx.doi.org/10.5194/angeo-32-181-2014

[29] Futatani, S., Horton, W., Kahlon, L.Z. and Kaladze, T.D. (2015) Rossby-Khantadze Electromagnetic Planetary Waves Driven by Sheared Zonal Winds in the E-layer Ionosphere. Physics of Plasmas, 22, 012906 (1-7).

[30] Kahlon, L.Z. and Kaladze, T.D. (2015) Generation of Zonal Flow and Magnetic Field in the Ionospheric E-layer. Journal of Plasma Physics, 81, 905810512 (1-12). 